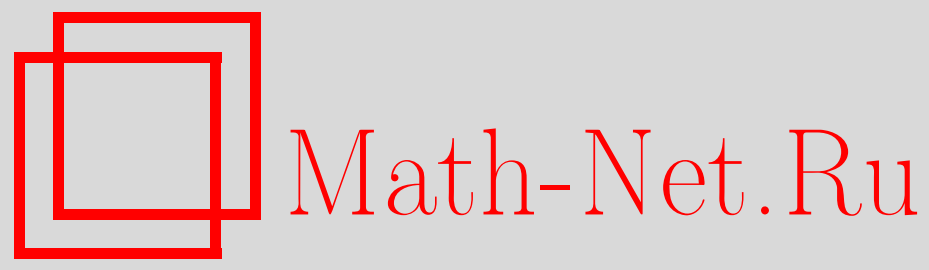

П. Е. Пушкарь, Индекс Маслова и симплектические теоремы Штурма, Функи. анализ и его прил., 1998, том 32, выпуск 3, 35-49

DOI: https://doi.org/10.4213/faa421

Использование Общероссийского математического портала Math$\mathrm{Net.Ru}$ подразумевает, что вы прочитали и согласны с пользовательским соглашением

http://www . mathnet.ru/rus/agreement

Параметры загрузки:

IP : 54.198 .64 .247

26 апреля 2023 г., 07:32:22

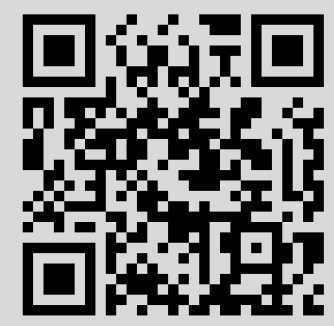


Функииональньй анализ и еәо приложения

1998, т. 32, вып. 3, с. 35-49

УДК 517.9

\section{Индекс Маслова и симплектические теоремы Штурма}

(C) 1998. П. Е. ПушкАРЬ

\section{Введение}

Теоремы Штурма о нулях решений дифференциального уравнения второго порядка описывают вращение прямой на фазовой плоскости этого уравнения $[3,13]$. В симплектической версии этих теорем вместо прямых рассматриваются лагранжевы плоскости, а вместо моментов пересечения с выделенной прямой - моменты нетрансверсальности с данной плоскостью [2]. Шлейф данной лагранжевой плоскости - гиперповерхность (с особенностями) в лагранжевом грассманиане, состоящая из всех лагранжевых плоскостей, не трансверсальных данной.

Индексом Маслова называется индекс пересечения кривой на лагранжевом грассманиане со шлейфом. Симплектические теоремы Штурма [2] описывают свойства индекса Маслова. Симплектическая теория Штурма была построена в работах Морса [10, 11], Лидского [9] и Арнольда [2], а ее эрмитов вариант в работах Ботта [4] и Эдвардса [5]. Свойства (не)осцилляционности гамильтоновых уравнений и коциклы, представляющие образующую первых гомологий симплектической группы, изучались Якубовичем [15-17]. Симплектическая теория Штурма применялась Гивенталем [7]. Им доказано свойство лагранжевой неосцилляционности уравнения Пикара-Фукса для гиперэллиптических интегралов. В работе определяется класс гиперповерхностей в лагранжевом грассманиане, на которые переносятся симплектические теоремы Штурма. Автор благодарен В. И. Арнольду и А. Г. Хованскому за полезные обсуждения.

\section{§1. Необходимые определения}

В этом параграфе строятся трансверсально ориентированные гиперповерхности в лагранжевом многообразии Грассмана, определяющие одномерный коцикл, совпадающий с индексом Маслова.

Рассмотрим симплектическое линейное пространство $\left(\mathbb{R}^{2 n}, \omega\right)$. Обозначим через $\Lambda_{n}$ многообразие лагранжевых подпространств в $\left(\mathbb{R}^{2 n}, \omega\right)$, называемое лагранжевым многообразием Грассмана. Зафиксируем координаты Дарбу $(p, q)=\left(p_{1}, \ldots, p_{n}, q_{1}, \ldots, q_{n}\right)$ в $\left(\mathbb{R}^{2 n}, \omega\right), \omega=\sum d p_{i} \wedge d q_{i}=d p \wedge d q$. Будем называть плоскость $p=0 \quad q$-плоскостью, а плоскость $q=0 \quad p$-плоскостью.

* Работа частично поддержана грантом INTAS-96-0713 и Российским фондом фундаментальных исследований (проект 96-01-01104). 
Рассмотрим множество $X \subset \Lambda_{n}$ всех лагранжевых плоскостей, трансверсальных плоскости $p=0$. Множество $X$ - открытое всюду плотное подмножество в многообразии $\Lambda_{n}$ (его карта), диффеоморфное $\mathbb{R}^{n(n+1) / 2}$. Лагранжева плоскость, содержашаяся в $X$, отождествляется с симметрической матрицей: матрице $A$ соответствует плоскость $q=A p$.

ОПРЕДЕлЕНИЕ [2]. Шлейфом данной лагранжевой плоскости называется множество всех лагранжевых плоскостей, не трансверсальных данной. В карте $X$ шлейф $p$-плоскости задается уравнением $\operatorname{det} A=0$.

Будем отождествлять элементы касательного пространства в точке линейного пространства и инвариантные относительно сдвигов векторные поля в линейном пространстве с элементами линейного пространства. В нашем случае мы отождествляем инвариантные относительно сдвигов векторные поля в карте $X$ с матрицами.

Рассмотрим гиперповерхность в $X$, заданную уравнением

$$
\left(L_{A_{1}} \cdots L_{A_{k}} \operatorname{det}\right)(A)=0,
$$

где $A_{1}, \ldots, A_{k}$ - положительно определенные матрицы $(0 \leqslant k<n), L_{v}$ производная вдоль векторного поля $v$ и det - определитель.

ОПРЕДЕЛЕНИЕ. Замыкание гиперповерхности $\left\{A:\left(L_{A_{1}} \cdots L_{A_{k}} \operatorname{det}\right)(A)=0\right\}$ $\subset X$ в лагранжевом многообразии Грассмана $\Lambda_{n}$ назовем обобщенныл шлей$\oint о м$ и обозначим его через $\Sigma_{\mathscr{A}}$. Иногда будем обозначать обобщенный шлейф через $\Sigma_{A_{1}, \ldots, A_{k}}$; при этом мы предполагаем, что заранее зафиксированы некоторые координаты Дарбу.

Таким образом, обобщенный шлейф строится по следующим данным: координатам Дарбу в $\left(\mathbb{R}^{2 n}, \omega\right)$ и $k(0 \leqslant k<n)$ положительно определенным $n \times n$-матрицам.

ЗАмечание. Этот набор данных шире, чем нужно: достаточно вместо координат Дарбу зафиксировать $k+2$ лагранжевых плоскостей, на которые наложены следующие условия:

1) первая плоскость трансверсальна остальным;

2) если выбраны какие-нибудь координаты Дарбу, такие, что первая плоскость является $q$-плоскостью, а вторая есть $p$-плоскость, то $k$ оставшихся лагранжевых плоскостей должны изображаться в соответствующей карте $X$ положительно определенными матрицами.

После этого повторим предыдущее определение обобщенного шлейфа с этими координатами Дарбу и получившимися матрицами. Получающаяся при этом гиперповерхность и выполнение условия 2) не зависят от произвола в выборе координат Дарбу.

Шлейф лагранжевой плоскости — частный случай обобщенного шлейфа $(k=0)$. Как будет показано ниже, при $n>2$ все обобщенные шлейфы в $\Lambda_{n}$ - гиперповерхности с особенностями. При $n=2$ обобшенный шлейф, построенный по одной матрице (первый нетривиальный пример обобщенного шлейфа), есть гладкая гиперповерхность в $\Lambda_{2}$, диффеоморфная двумерной cфepe. 
ОПРЕДЕЛЕНИЕ [2]. Положительными векторами на лагранжевом многообразии Грассмана называются векторы скоростей движения лагранжевых плоскостей под действием положительно определенных гамильтонианов.

Шлейф любой плоскости трансверсально ориентирован положительными векторами в неособых точках. Индекс пересечения ориентированных петель со шлейфом определяет каноническую образуюшую группы $H^{1}\left(\Lambda_{n}, \mathbb{Z}\right)=\mathbb{Z}$ (см. [1, 2]) — класс Маслова.

Обобщенный шлейф - гиперповерхность (с особенностями) в $\Lambda_{n}$. Свойства обобщенного шлейфа во многом похожи на свойства шлейфа лагранжевой плоскости. Верна следующая теорема:

ТЕОРема 1.1. Обобщенньй шлей $\Sigma_{\mathscr{A}}$ - гиперповерхность в лагранжевом многообразии Грассмана $\Lambda_{n}$, такая, что:

1) положительные векторы трансверсальны $\kappa \Sigma_{\mathscr{A}}$ в неособых точках;

2) трансверсальная ориентация обобшенного шлейфа положительньми векторами задает элемент $\alpha_{\mathscr{A}} \in H^{1}\left(\Lambda_{n}, \mathbb{Z}\right)$ - индекс пересечения ориентированных петель с $\Sigma_{\mathscr{A}}$;

3) элемент $\alpha_{\mathscr{A}}$ совпадает с классом Маслова.

ОПРЕДЕЛЕНИЕ. Будем называть положительным nутем гладкий путь в лагранжевом многообразии Грассмана, вектор скорости которого в любой точке положителен.

СЛЕдСТВИЕ 1.2. Индекс пересечения положительного пути с обобщенным шлейфом неотрицателен.

Доказательство теоремы 1.1 приводится в 3 . Пересечение обобщенного шлейфа с картой $X$ является нулевой поверхностью уровня гиперболического полинома. Необходимые нам утверждения о гиперболических полиномах собраны в следующем параграфе.

\section{§2. Гиперболические полиномы}

Напомним, что вешественный полином степени $n$ называется гиперболическим вдоль вектора $a$, если его ограничение на любую прямую, параллельную $a$, имеет ровно $n$ вещественных корней (с учетом кратностей). В этом случае будем называть вектор а гиперболическим вектором данного полинома или, допуская вольность речи, просто аиперболическим.

Векторы, вдоль которых данный однородный полином гиперболичен, образуют открытые выпуклые конусы [6, 14]. Нам понадобятся следующие утверждения об однородных гиперболических полиномах.

Пусть $K$ - открытый конус, образующий связную компоненту области гиперболических векторов однородного полинома $f$.

ПРЕДЛОЖЕНИЕ 2.1 (см. [2]). Конус $K$, параллельно перенесенный в точку поверхности $f=0$, не пересекается с этой поверхностью в достаточно малой окрестности перенесенной вершинь.

Как и в $\S 1$, будем отождествлять инвариантные относительно сдвигов (постоянные) векторные поля на линейном пространстве с векторами этого пространства. 
ПРЕДЛОЖЕНИЕ 2.2 (см. [14]). Производная полинома $f$ вдоль постоянного гиперболического векторного поля, равного вектору из конуса $K$, лвляется однородным гиперболическим полиномом относительно векторов из конуса $K$.

ПРЕДЛОЖЕНИЕ 2.3. Путь, вектор скорости которого в любой момент пересечения с поверхностью $f=0$ принадлежит конусу $K$, пересекает эту поверхность конечное число раз.

ДокАЗАТЕльСТво. Обозначим через $g(x)$ число неположительных корней (с учетом кратностей) полинома $f(x+t a)$ от переменной $t, a \in K$. Функция $g$ локально постоянна на дополнении к гиперповерхности $f=0$, так как $f$ гиперболичен. Ее значение не зависит от выбора $a \in K$. Ограниченная на путь, она возрастает при пересечении пути с $f=0$ - это легко вывести из предложения 2.1. Предложение доказано.

ПРЕДЛОЖЕНИЕ 2.4. Множество критических точек производной однородного полинома $f$ вдоль постолнного гиперболического векторного поля содержится в множестве критических точек полинома $f$.

ДоказАтельство. Обозначим через $a \in K$ постоянное гиперболическое поле. Пусть $x$ - критическая точка гиперболического полинома $L_{a} f$. Поскольку полином $L_{a} f$ однородный, $\left(L_{a} f\right)(x)=0$. Кратность корня $t=0$ полинома $\left(L_{a} f\right)(x+t b)$ от переменной $t$ постоянна при $b \in K$, согласно предложениям 2.1 и 2.2 , так как все корни полинома $\left(L_{a} f\right)(x+t b)$ от переменной $t$ вещественны и непрерывно зависят от вектора $b \in K$. Таким образом, точка $x$ является критической точкой гиперболического полинома $L_{a} f$, если и только если $t=0$ - кратный корень полинома $l(t)=\left(L_{a} f\right)(x+t a)$. Полином $l(t)$ равен производной полинома $f(x+t a)$ от переменной $t$. Полиномы $f(x+t a)$ и $l(t)$ от переменной $t$ гиперболические. (Напомним, что вещественный полином от одной переменной называется гиперболическим, если все его корни вещественны.) По лемме Ролля корень производной гиперболического полинома является кратным, если и только если он - кратный корень (кратности не меньше 3) самого полинома. Следовательно, $t=0-$ кратный корень полинома $f(x+t a)$ от $t$ и $x$ - критическая точка полинома $f$. Доказательство закончено.

Следующее утверждение хорошо известно. Это следствие теоремы о приведении пары форм, одна из которых положительно определена, к главным осям.

ПредлОжениЕ 2.5. Определитель симметрической матрицы - гиперболический однородный полином на пространстве симметрических матрич относительно конуса положительно определенных матрич.

СлЕДСТвиЕ 2.6. Пусть $A_{1}, \ldots, A_{k}$ - положительно определенные матричы. Полином $L_{A_{1}} \cdots L_{A_{k}} \operatorname{det}$ - гиперболический однородный полином на пространстве симметрических матрии относительно конуса положительно определенных матрич.

\section{§3. Свойства обобщенного шлейфа}

Напомним, что через $X$ мы обозначили карту многообразия $\Lambda_{n}$, состоящую из всех лагранжевых плоскостей, трансверсальных $q$-плоскости. При 
помощи линейной структуры мы отождествляем касательные векторы в разных точках множества $X$, постоянные векторные поля на $X$ и матрицы элементы множества $X$.

ЛЕмма 3.1. Положительнье векторы в карте $X$ лагранжева многообразия Грассмана изображаются положительно определенньми матрииами.

ДокАЗАтЕльство. Пусть $H$ - положительно определенный гамильтониан. Рассмотрим лагранжеву плоскость $q=A p$. Ее образ под действием фазового потока гамильтонова векторного поля $\dot{p}=-H_{q}, \dot{q}=H_{p}$ с гамильтонианом $H$ за время $\varepsilon$ есть плоскость $q=(A+\varepsilon B) p+o(\varepsilon)$. Докажем, что матрица $B$ положительно определена. Действительно,

$$
A p+\varepsilon H_{p}(p, A p)=(A+\varepsilon B)\left(p-\varepsilon H_{q}(p, A p)\right)+o(\varepsilon),
$$

откуда $H_{p}(p, A p)=B p-A H_{q}(p, A p)$. Следовательно,

$$
\begin{aligned}
\langle B p, p\rangle & =\left\langle H_{p}(p, A p), p\right\rangle+\left\langle A H_{q}(p, A p), p\right\rangle \\
& =\left\langle H_{p}(p, A p), p\right\rangle+\left\langle H_{q}(p, A p), A p\right\rangle=2 H(p, A p)
\end{aligned}
$$

по теореме Эйлера. Значит, матрица $B$ положительно определена. Верно и обратное: любая положительно определенная матрица изображает положительный вектор. Лемма доказана.

Рассмотрим карту $Y$ лагранжева грассманиана $\Lambda_{n}$, состоящую из лагранжевых плоскостей, трансверсальных плоскости $p=0$ ( $q$-плоскости). Как и в случае карты $X$, мы отождествляем в карте $Y$ лагранжеву плоскость с симметрической матрицей $B$ : матрице $B$ соответствует плоскость $p=B q$. Координаты плоскости $\lambda \in X \cap Y$ в картах $X$ и $Y$ связаны соотношением $A=B^{-1}(A-$ матрица, соответствующая плоскости $\lambda$ в карте $X, B-$ в карте $Y)$.

ЛЕмма 3.2. а) $B$ карте $Y$ уравнение $\left(L_{A_{1}} \cdots L_{A_{k}} \operatorname{det}\right)(A)=0$ вьглядит $\kappa a \kappa\left(L_{-B A_{1} B} \cdots L_{-B A_{k} B} \frac{1}{\mathrm{det}}\right)(B)=0$.

б) $\left(L_{-B A_{1} B} \cdots L_{-B A_{k} B} \frac{1}{\operatorname{det}}\right)(B)=P_{A_{1}, \ldots, A_{k}}(B) / \operatorname{det} B$, zде $P_{A_{1}, \ldots, A_{k}}(B)$ однородныий полином степени $k$, равньй сумме слагаемых вида

$$
\pm \operatorname{tr}\left(A_{i_{1}} B \cdots A_{i_{m_{1}}}\right) \operatorname{tr}\left(A_{i_{m_{1}+1}} B \cdots A_{i_{m_{2}}} B\right) \cdots \operatorname{tr}\left(A_{i_{m_{l-1}+1}} B \cdots A_{i_{m_{l}}} B\right)
$$

$\left(\left(i_{1}, \ldots, i_{m_{l}}\right)\right.$ - перестановка элементов $\left.(1, \ldots, k)\right)$.

ДокАЗАТЕльство. а) Для любой матрицы $M(\operatorname{det} M \neq 0)$ выполняется равенство $\left(M+\varepsilon A_{i}\right)^{-1}=M^{-1}-\varepsilon M^{-1} A_{i} M^{-1}+o(\varepsilon)$.

б) Легко проверить, что $\left(L_{-B A_{1} B} \frac{1}{\operatorname{det}}\right)(B)=\operatorname{tr} A_{1} B / \operatorname{det} B$. Далее доказательство проводится по индукции. Например, $P_{A_{1}, A_{2}}(B)=\operatorname{tr} A_{1} B \operatorname{tr} A_{2} B-$ $\operatorname{tr} A_{1} B A_{2} B$. Доказательство закончено.

Для удобства будем иногда обозначать оператор $L_{A_{1}} \cdots L_{A_{k}}$ через $L_{\mathscr{A}}$, a полином $P_{A_{1}, \ldots, A_{k}}$ через $P_{\mathscr{A}}$.

Лемма 3.3. Множество особих точек гиперповерхности $L_{\mathscr{A}} \operatorname{det}=0$ имеет коразмерность, не меньшую, чем 3 , в карте $X$.

Множество особих точек гиперповерхности $\left\{B: P_{\mathscr{A}}(B)=0\right\}$ имеет коразмерность, не меньшую, чем 3 , в карте $Y$, причем гиперповерхность 
$\left\{B: P_{\mathscr{A}}(B)=0\right\}$ пересекается с гиперповерхностью $\{B: \operatorname{det} B=0\}$ трансверсально вне множества коразмерности, не меньшей, чем 3 , в карте $Y$.

ДокАЗАТЕльство. Первое утверждение леммы следует из предложения 2.4, поскольку множество особых точек гиперповерхности $L_{\mathscr{A}} \operatorname{det}=0$ содержится в множестве особых точек гиперповерхности $\operatorname{det}=0$, коразмерность которого равна 3. Докажем второе утверждение. Пересечение множества особых точек гиперповерхности $\left\{B: P_{\mathscr{A}}(B)=0\right\}$ и множества $\{B: \operatorname{det} B \neq 0\}$ имеет коразмерность, не меньшую, чем 3 , в карте $Y$, поскольку это изображение множества особых точек гиперповерхности $L_{\mathscr{A}} \operatorname{det}=0$ в карте $Y$.

Множество особых точек гиперповерхности $\{B: \operatorname{det} B=0\}$ имеет коразмерность 3 в карте $Y$. Следовательно, достаточно показать, что гиперповерхность $\left\{B: P_{\mathscr{A}}(B)=0\right\}$ и многообразие неособых точек гиперповерхности $\{B: \operatorname{det}(B)=0\}$ пересекаются трансверсально вне множества коразмерности по меньшей мере 3 в $Y$.

Множество неособых точек гиперповерхности $\{B: \operatorname{det}(B)=0\}-$ это множество матриц с одномерным ядром. Оно расслоено над соответствующим проективным пространством (матрице соответствует ядро). Докажем, что множество особых корней уравнения $P_{\mathscr{A}}(B)=0$, ограниченного на любой слой этого расслоения, имеет коразмерность не меньше 2 в слое. Из этого вытекает второе утверждение леммы.

Рассмотрим слой этого расслоения над прямой $q_{1}=\cdots=q_{n-1}=0$. Правый столбец и нижняя строка матриц из этого слоя нулевые, а определитель левого верхнего минора размера $(n-1) \times(n-1)$ отличен от нуля. Обозначим этот минор через $\widetilde{B}$. Уравнение $P_{\mathscr{A}}(B)=P_{A_{1}, \ldots, A_{k}}(B)=0$ в этом слое выглядит так: $P_{\tilde{A}_{1}, \ldots, \tilde{A}_{k}}(\widetilde{B})=0$ (по лемме 3.2 ), где $\tilde{A}_{i}$ - левый верхний минор размера $(n-1) \times(n-1)$ матрицы $A_{i}$. Матрицы $\tilde{A}_{i}$ положительно определены; следовательно, как показано выше, уравнение $P_{\mathscr{A}}(B)=0$ при $k<n-1$ определяет в рассматриваемом слое гиперповерхность, множество особых точек которой имеет в слое коразмерность, не меньшую, чем 3 . Если $k=n-1$, то $P_{\tilde{A}_{1}, \ldots, \tilde{A}_{k}}(\widetilde{B}) / \operatorname{det} \widetilde{B}=\left(L_{\tilde{A}_{1}} \cdots L_{\tilde{A}_{k}} \operatorname{det}\right)\left(\widetilde{B}^{-1}\right) \neq 0$. Значит, рассматриваемый слой не содержится в гиперповерхности $\left\{B: P_{\mathscr{A}}(B)=0\right\}$. Случай других слоев сводится заменой координат Дарбу, сохраняющих $p$ - и $q$-плоскости, к рассмотренному. Лемма доказана.

Обозначим объединение множеств особых корней уравнения $P_{A_{1}, \ldots, A_{k}}(B)$ $=0$ на слое расслоения, рассмотренного в доказательстве леммы 3.3 , по всем слоям этого расслоения через $O$. Коразмерность множества $O$ равна по меньшей мере 3 согласно лемме 3.3.

СлЕДСТВиЕ 3.4. Пересечение обобщенного шлейба с объединением карт $X и Y$ - аиперповерхность, гладкая вне множества коразмерности, не меньшей, чем 3 .

ДокАЗАТЕльство. Действительно, пересечение обобщенного шлейфа с картой $X$ неособо вне множества коразмерности, не меньшей, чем 3 , - это первое утверждение предыдущей леммы. Особые точки пересечения обобщенного шлейфа с картой $Y$, принадлежащие $Y \backslash X$, содержатся в множестве особых точек гиперповерхности $\{B: \operatorname{det}(B)=0\}$ и множестве $O$. 
ЛЕммА 3.5. Положительнье векторь трансверсальнь обобщенному шлейфу в его неособъх точках.

ДокАЗАтЕльство. Пусть лагранжева плоскость $\lambda$ - неособая точка обобщенного шлейфа. Тогда $\lambda$ - предел последовательности неособых точек пересечения обобщенного шлейфа с картой $X$. В неособых точках пересечения обобшенного шлейфа с картой $X$ положительные векторы трансверсальны обобщенному шлейфу согласно утверждению 2.1 и лемме 3.1. Выберем координаты Дарбу $(\tilde{p}, \tilde{q})$, такие, что $\lambda$ есть $\tilde{p}$-плоскость. В карте $Z$ лагранжевых плоскостей, трансверсальных $\tilde{q}$-плоскости, положительные векторы, согласно лемме 3.1, изображаются положительно определенными матрицами. Лемма вытекает из следующего простого утверждения:

ПРЕДЛОЖЕНИЕ 3.6. Пусть $K$ - открытый конус в $\mathbb{R}^{N}$ u $Г$ - әладкая гиперповерхность в $\mathbb{R}^{N}$. Тогда множество точек гиперповерхности $\Gamma, ~ в$ которых все векторы из $K$ трансверсальны $Г$, замкнуто в $Г$.

ДоказАтельство. Действительно, множество точек из $Г$, таких, что найдется вектор из $K$, касающийся $\Gamma$ в этой точке, очевидно, открыто в $\Gamma$.

ЛЕммА 3.7. Индекс пересечения ориентированных замкнутых кривых $c$ обобшенным шлейфом $\Sigma_{\mathscr{A}}=\Sigma_{A_{1}, \ldots, A_{k}}$ (трансверсально ориентированньл положительными векторами) определяет элемент $\widetilde{\alpha}_{A_{1}, \ldots, A_{k}} \in H^{1}(X \cup Y, \mathbb{Z})$. Этот элемент не зависит от выбора положительно определенньх матрии $A_{1}, \ldots, A_{k}$.

ДокАЗАтЕльство. Действительно, первое утверждение леммы вытекает из следствия 3.4 и леммы 3.5. Докажем второе утверждение. Рассмотрим петлю в $X \cup Y$ и приведем ее в общее положение по отношению к $\Sigma_{A_{1}, \ldots, A_{k}}$ и шлейфу $q$-плоскости. Полученная петля пересекает $\Sigma_{A_{1}, \ldots, A_{k}}$ и шлейф $q$-плоскости трансверсально в неособых точках этих гиперповерхностей, причем, согласно лемме 3.3, можно считать, что точки пересечения петли с $\Sigma_{A_{1}, \ldots, A_{k}}$ содержатся в карте $X$. Если матрицы $B_{1}, \ldots, B_{k}$ близки к матрицам $A_{1}, \ldots, A_{k}$ соответственно, то полученная петля пересечет $\Sigma_{B_{1}, \ldots, B_{k}}$ трансверсально в близких точках. Докажем это.

Пусть $\gamma(t), t \in \mathbb{R} / \mathbb{Z}$, - полученная петля, а $t_{1}, \ldots, t_{l}$ - моменты пересечения этой петли со шлейфом $q$-плоскости. Тогда при достаточно малом $\varepsilon$ пути $\gamma(t), t \in\left[t_{j}-\varepsilon, t_{j}+\varepsilon\right]$, содержатся в карте $Y$ и не пересекаются с гиперповерхностью $\left\{B: P_{A_{1}, \ldots, A_{k}}(B)=0\right\}$. Следовательно, эти пути не пересекаются с гиперповерхностью $\left\{B: P_{B_{1}, \ldots, B_{k}}(B)=0\right\}$ (если матрицы $B_{1}, \ldots, B_{k}$ близки к матрицам $\left.A_{1}, \ldots, A_{k}\right)$, поскольку уравнение $P_{A_{1}, \ldots, A_{k}}(B)=0$ непрерывно зависит от $A_{1}, \ldots, A_{k}$. Тем более пути $\gamma(t), t \in\left[t_{j}-\varepsilon, t_{j}+\varepsilon\right]$, не пересекаются с $\Sigma_{B_{1}, \ldots, B_{k}}$.

С другой стороны, пути $\gamma(t), t \in\left[t_{j}+\varepsilon, t_{j+1}-\varepsilon\right]$, содержатся в карте $X$. Уравнение $L_{A_{1}} \cdots L_{A_{k}}(A)=0$ гладко зависит от $A_{1}, \ldots, A_{k} ;$ следовательно, пути $\gamma(t), t \in\left[t_{j}+\varepsilon, t_{j+1}-\varepsilon\right]$, пересекут $\Sigma_{B_{1}, \ldots, B_{k}}$ трансверсально в близких точках по теореме о неявной функции. Остается заметить, что вклад каждой точки пересечения в индекс пересечения не меняется при малом изменении матриц $A_{1}, \ldots, A_{k}$. Мы доказали, что элемент $\widetilde{\alpha}_{A_{1}, \ldots, A_{k}}$ локально постоянен; следовательно, он постоянен, так как положительно определенные матрицы образуют связное множество. Лемма доказана. 
Определим на карте $X$ функцию $g_{\mathscr{A}}$ следуюшим образом: $g_{\mathscr{A}}(B)$ равно числу неположительных корней полинома $L_{\mathscr{A}}(B+t P)$ от переменной $t(P-$ какая-нибудь положительно определенная матрица). Функция $g_{\mathscr{A}}$ не зависит от участвующей в ее определении матрицы $P$. Из результатов $\S 2$ легко выводится следующее

ПРЕДЛОЖЕНИЕ 3.8. Индекс пересечения пути, содержашегося в карте $X$, c $\Sigma_{\mathscr{A}}$ равен прирашению функиии $g_{\mathscr{A}}$. Если начало такого пути - отрицательно определенная, а конеч - положительно определенная матриць, то его индекс пересечения $c \Sigma_{\mathscr{A}}$ равен $n-k$.

Обозначим через $E$ единичную матрицу, а через $\Sigma_{E}^{k}$ обобщенный шлейф $\Sigma_{A_{1}, \ldots, A_{k}}$ с $A_{1}=\cdots=A_{k}=E$.

ЛЕмма 3.9. Обобиенный шлей $\Sigma_{E}^{k}$ - гиперповерхность, гладкал вне множества коразмерности по меньшей мере 3 в $\Lambda_{n}$. Индекс пересечения ориентированных замкнутых кривых с $\Sigma_{E}^{k}$ (трансверсально ориентированньм положительными векторами) определяет элемент в $H^{1}\left(\Lambda_{n}, \mathbb{Z}\right)$, совпадающий с классом Маслова.

ДокАЗАТЕльство. Если $k=0$, то наш обобщенный шлейф есть просто шлейф $p$-плоскости и утверждение леммы доказано в [1]. Пусть $k>0$. Докажем, что $\Sigma_{E}^{k}$ пересекается с $\Lambda_{n} \backslash(X \cup Y)$ по множеству коразмерности по меньшей мере 3 (для $k=0$ последнее неверно). Из этого ввиду следствия 3.4 вытекает первое утверждение леммы.

Очевидно такое утверждение:

ПРЕДЛОЖЕНИЕ 3.10. Пусть $A_{1}=\cdots=A_{k}=E$ u $\mu_{1}, \ldots, \mu_{n}-$ собственнье числа симметрической матричь $M$. Тогда

$$
L_{\mathscr{A}} \operatorname{det}(M)=k ! \sum_{i_{1}<\cdots<i_{k}} \mu_{i_{1}} \cdots \mu_{i_{k}}, \quad P_{\mathscr{A}}(M)=k ! \sum_{i_{1}<\cdots<i_{n-k}} \mu_{i_{1}} \cdots \mu_{i_{n-k}} .
$$

Продолжим доказательство леммы 3.9. Множество $\Lambda_{n} \backslash(X \cup Y)$ является подмногообразием (с особенностями) коразмерности 2 в $\Lambda_{n}$. Множество лагранжевых плоскостей, пересекающихся с $p$ - и $q$-плоскостями по одномерному подпространству, образует открытое подмногообразие $L$ коразмерности 2 в $\Lambda_{n}$ (например, при $n=2$ это незаузленная окружность в $\Lambda_{2}$ ). Дополнение до $L$ в $\Lambda_{n} \backslash(X \cup Y)$ образует подмногообразие (с особенностями) коразмерности, не меньшей, чем 3 , в $\Lambda_{n}$. Плоскости $\lambda \in L$ соответствует набор из $n-2$ ненулевых «собственных чисел» - нужно рассмотреть последовательность плоскостей (матриц) из $X$, сходящихся к $\lambda$, и взять пределы собственных чисел. Один из этих пределов равен нулю и один - бесконечности, а остальные образуют нужный нам набор. Пользуясь предложением 3.10, легко проверить, что условие принадлежности плоскости $\lambda$ обобщенному шлейфу $\Sigma_{E}^{k}$ - нетривиальное условие на этот набор. Следовательно, $\Sigma_{E}^{k}$ пересекает $L$ по гиперповерхности. Первое утверждение леммы доказано.

Докажем второе утверждение леммы. Трансверсально ориентированный положительными векторами обобщенный шлейф $\Sigma_{E}^{k}$ определяет элемент $\alpha_{k E}$ в $H^{1}\left(\Lambda_{n}, \mathbb{Z}\right)$, поскольку множество его особых точек имеет коразмерность 3 в $\Lambda_{n}$. Докажем, что $\alpha_{k E}$ совпадает с классом Маслова. 
Поскольку $\pi_{1}\left(\Lambda_{n}\right)=\mathbb{Z}[1]$, достаточно показать, что значения класса $\alpha_{k} E$ и класса Маслова на какой-нибудь нестягиваемой петле совпадают. Рассмотрим петлю $\gamma$, образованную следующими путями $\gamma_{1}$ и $\gamma_{2}$. Путь $\gamma_{1}(t)=$ $(t-1) A+t B, t \in[0,1]$, содержится в карте $X$, а путь $\gamma_{2}(t)=(1-t) B^{-1}-t A^{-1}$, $t \in[0,1]$, - в карте $Y$, причем $A$ и $B$ - положительно определенные матрицы. Концы путей $\gamma_{1}$ и $\gamma_{2}$ не принадлежат $\Sigma_{E}^{k}$. Индекс Маслова петли $\gamma$ равен $n$, поскольку путь $\gamma_{2}$ не пересекает шлейфа $p$-плоскости, а индекс пересечения пути $\gamma_{1}$ со шлейфом $p$-плоскости равен $n$ [2]. Следовательно, индекс пересечения петли $\gamma$ с $\Sigma_{E}^{k}$ делится на $n$. Индекс пересечения пути $\gamma_{1}$ с $\Sigma_{E}^{k}$ равен $n-k$, согласно предложению 3.8. Индекс пересечения пути $\gamma_{2}$ с $\Sigma_{E}^{k}$, во-первых, неотрицателен, так как путь $\gamma_{2}$ положителен (заметим, что положительные векторы изображаются в карте $Y$ отрицательно определенными матрицами), и, во-вторых, не превосходит $k$, поскольку пересечение $\Sigma_{E}^{k}$ с картой $Y$ содержится в нулевой поверхности уровня полинома $P_{E}, \ldots, E$, степень которого равна $k$. Таким образом индекс пересечения петли $\gamma$ с $\Sigma_{E}^{k}$ положителен и не больше $n$; следовательно, он равен $n$. Лемма 3.9 доказана.

ЛЕмма 3.11. Индекс пересечения ориентированных замкнутых кривых $c$ $\Sigma_{\mathscr{A}}($ трансверсально ориентированньм положительньми векторами) определяет элемент в $H^{1}\left(\Lambda_{n}, \mathbb{Z}\right)$, совпадаюший с классом Маслова.

ДокАЗАтЕльство. Возьмем петлю в $\Lambda_{n}$ и пошевелим ее так, чтобы она оказалась в $X \cup Y$. Значение $\widetilde{\alpha}_{A_{1}, \ldots, A_{k}}$ на получившейся петле равно значению $\widetilde{\alpha}_{E, \ldots, E}$ по лемме 3.7. По лемме 3.9 оно совпадает с индексом Маслова. Следовательно, индекс пересечения корректно определен и лемма доказана.

ДокАЗАТЕльСТво ТЕоремы 1.1. Теорема 1.1 доказана в леммах 3.5 и 3.11.

ЗАмечАния. 1) Пересечение обобщенного шлейфа $\Sigma_{\mathscr{A}}$ с картой $Y$ совпадает с поверхностью $\left\{B: P_{\mathscr{A}}(B)=0\right\}$. Полином $P_{\mathscr{A}}$ является гиперболическим относительно конуса положительно определенных матриц. Эти утверждения легко выводятся из того, что индекс пересечения пути $\gamma_{2}$, рассмотренного в доказательстве леммы 3.9 , с $\Sigma_{\mathscr{A}}$ равен $k$.

2) Доказательство теоремы 1.1 можно было бы провести иначе, если показать, что особенности обобщенного шлейфа имеют коразмерность, не меньшую, чем 3 , в лагранжевом грассманиане. Доказательство этого факта автору неизвестно.

\section{§4. Построение коцикла}

Конус положительных векторов соотносится с обобщенным шлейфом так же, как конус векторов гиперболичности полинома $f$ соотносится с поверхностью $f=0$ (см. предложение 2.1). Это обстоятельство позволяет определить на $\Lambda_{n}$ одномерный коцикл. Для разных целей полезно использовать индекс пересечения незамкнутых путей с (обобщенным) шлейфом. Для шлейфа лагранжевой плоскости такой индекс определен в [12].

ЛЕммА 4.1. Моменты пересечения положительного пути со шлейфом любой лагранжевой плоскости $\lambda$ изолированы.

ДокАЗАтЕльство. Пусть $\gamma(t), t \in] 0,1\left[\right.$, - положительный путь и $\gamma\left(t_{0}\right)$ принадлежит шлейфу плоскости $\lambda$. 
Можно выбрать координаты Дарбу $(\tilde{p}, \tilde{q})$ таким образом, что плоскость $\lambda$ - это $\tilde{p}$-плоскость, а плоскость $\gamma\left(t_{0}\right)$ трансверсальна $\tilde{q}$-плоскости. В карте многообразия $\Lambda_{n}$ плоскостей, трансверсальных $\tilde{q}$-плоскости, шлейф плоскости $\lambda$ задается уравнением $\operatorname{det}(A)=0$. Применяя предложения 2.3 и 2.5 и лемму 3.1 , завершаем доказательство.

ЛЕмма 4.2. Моменты пересечения положительного пути с обобщенным шлейбом изолированы.

ДокаЗАтЕльство. Моменты пересечения положительного пути со шлейфом $q$-плоскости изолированы. Следовательно, путь разбивается на конечное число кусков, содержащихся в карте $X$. На каждом таком куске число моментов пересечения с обобщенным шлейфом конечно согласно 2.6, 2.3 и 3.1. Лемма доказана.

Рассмотрим координаты Дарбу $(\tilde{p}, \tilde{q})$ и карту $X_{\tilde{p}}$ грассманиана $\Lambda_{n}$, состоящую из всех лагранжевых плоскостей, трансверсальных $\tilde{q}$-плоскости. Как и выше, мы отождествляем лагранжевы плоскости из $X_{\tilde{p}}$ с симметрическими матрицами.

ТеОрема 4.3. Конус положительно определенных матрии в карте $X_{\tilde{p}}$, параллельно перенесенный в точку обобщенного шлейфа, не пересекается $c$ обобшенным шлейфом в достаточно малой окрестности перенесенной вершиньл.

ДокАЗАТЕльство. Рассмотрим точку $x_{0} \in X_{\tilde{p}}$ обобщенного шлейфа и проведем через нее положительный путь (в карте $X_{\tilde{p}}$ ), пересекающий обобщенный шлейф только в точке $x_{0}$. Это можно сделать в силу леммы 4.2. Если бы утверждение теоремы было неверно, то нашелся бы другой положительный путь (в карте $X_{\tilde{p}}$ ) с теми же концами, что и первый, проходящий через точку $x_{0}$ и сколь угодно много неособых точек обобщенного шлейфа. Индексы пересечения этих путей с обобщенным шлейфом совпадают, так как эти пути гомотопны (с закрепленными концами). Следовательно, индекс пересечения первого пути сколь угодно велик (см. следствие 1.2). Противоречие.

ЗАмЕчАниЕ. Аналогичное утверждение верно для конуса отрицательно определенных матриц.

Шлейф лагранжевой плоскости естественным образом стратифицирован размерностью пересечения с ней. Подобная стратификация имеется и для обобщенного шлейфа.

Пусть $x_{0}$ - точка обобщенного шлейфа. Проведем через нее (короткий) положительный путь $\gamma$, не пересекаюший обобщенный шлейф в других точкаx.

ОПРЕДЕЛЕНИЕ. Назовем кратностью точки $x_{0}$ индекс пересечения пути $\gamma$ с обобщенным шлейфом.

ПРЕДЛОЖЕНИЕ 4.4. Кратность корректно определена и положительна.

ДокАЗАТЕльство. Рассмотрим два положительных пути $\gamma_{1}$ и $\gamma_{2}$, проходящие через точку $x_{0}$ обобщенного шлейфа (и больше не пересекающие обобщенный шлейф). Будем считать, что они помещаются в карте $X_{\tilde{p}}$ грассманиана $\Lambda_{n}$, связанной с координатами Дарбу $(\tilde{p}, \tilde{q})$. Путь $\gamma_{1}$ (в силу теоремы 4.3 и замечания) можно прогомотопировать в путь $\gamma_{2}$ так, что при 
гомотопии концы не будут пересекать обобщенный шлейф. Это доказывает корректность определения. Неособые точки плотны в обобшенном шлейфе, из чего следует вторая часть предложения.

ЗАмЕчАниЕ. Кратность точки $\beta$ шлейфа лагранжевой плоскости $\alpha$ равна размерности пересечения плоскостей $\alpha$ и $\beta$. При $n>2$ все обобщенные шлейфы в $\Lambda_{n}$ - гиперповерхности с особенностями, поскольку для обобщенного шлейфа $\Sigma_{\mathscr{A}}$, построенного по координатам Дарбу $(p, q)$ и матрицам $A_{1}, \ldots, A_{k}$, кратность $p$-плоскости равна $n-k$, а кратность $q$-плоскости равна $k$. Кратность неособой точки шлейфа равна 1 . Видимо, верно и утверждение, что если кратность равна 1, то точка неособа.

Пусть $\Sigma$ - обобщенный шлейф. Определим (аналогично [2, 8]) коцикл $\operatorname{ind}_{\Sigma}$, совпадающий на кривых с концами вне $\Sigma$ с индексом пересечения этих кривых с $\Sigma$.

Рассмотрим путь $\gamma(t), t \in[0,1]$, в $\Lambda_{n}$. Пусть $\gamma_{0}$ - отрицательный путь, входящий в начало $\gamma(0)$ пути $\gamma$, и $\gamma_{1}$ - положительный путь, выходящий из конца $\gamma(1)$ пути $\gamma$. Пути $\gamma_{0}$ и $\gamma_{1}$, согласно теореме 4.3 и замечанию, можно выбрать так, чтобы они могли пересекать обобщенный шлейф только в конце и начале соответственно.

ОПРЕДЕЛЕНИЕ. Положим $\operatorname{ind}_{\Sigma}(\gamma)$ равным индексу пересечения пути $\gamma_{0} \cup \gamma \cup \gamma_{1} \quad \mathrm{c} \quad \Sigma$.

ПРЕДЛОЖЕНИЕ 4.5. Число $\operatorname{ind}_{\Sigma}(\gamma)$ корректно определено. Функиия $\operatorname{ind}_{\Sigma}$ - коцикл. Когомологический класс коиикла ind $\mathrm{i}_{\Sigma}$ - класс Маслова.

ДокАЗАтЕльство. Корректность определения $\operatorname{ind}_{\Sigma}(\gamma)$ проверяется так же, как корректность определения кратности. Легко видеть, что ind $\Sigma$ коцепь. Тот факт, что ind $\Sigma$ - коцикл, следует из теоремы 1.1.

Легко проверяется справедливость следующего утверждения:

ПРЕДЛОЖЕНИЕ 4.6. Пусть $\gamma$ - положительный путь. Значение кочикла $\operatorname{ind}_{\Sigma}$ на пути $\gamma$ равно числу точек пересечения пути $\gamma$, за исключением начала этого пути, с обобшенным шлейфом $\Sigma$ с учетом кратностей, приписанных точкам из $\Sigma$.

Пусть $g_{H}^{\varepsilon}$ - отображение фазового потока гамильтонова векторного поля с положительно определенным (возможно, неавтономным) гамильтонианом $H$ за время $\varepsilon$.

ПРЕДЛОЖЕНИЕ 4.7. Пусть $\gamma(t), t \in[0,1],-$ путь в $\Lambda_{n}$. Значение коиикла ind $\operatorname{in}_{\Sigma}$ на пути $\gamma$ равно его значению на пути $g_{H}^{\varepsilon}(\gamma)$ при любом достаточно малом (зависящем от $H$ и $\gamma$ ) положительном $\varepsilon$.

ДокАЗАТЕльство. Действительно, при малом $\varepsilon$ путь, построенный при определении $\operatorname{ind}_{\Sigma}(\gamma)$, гомотопен в классе путей с концами вне $\Sigma$ пути $g_{H}^{\varepsilon}(\gamma)$.

\section{$\S 5$. Симплектические теоремы Штурма}

В этом параграфе доказываются обобщения симплектических теорем Штурма [2]. В наших рассуждениях особенно важна следующая простая лемма: 
ЛЕмма 5.1. Любые две трансверсальные лагранжевы плоскости в $\Lambda_{n}$ можно соединить положительным путем $\gamma$, таким, ито $0 \leqslant \operatorname{ind}_{\Sigma}(\gamma) \leqslant n$ для любого обобщенного илейфа $\Sigma$.

ДокАЗАТЕльство. Выберем координаты Дарбу в $\left(\mathbb{R}^{2 n}, \omega\right)$, такие, что первая плоскость совпадает с $p$-плоскостью, а вторая - с $q$-плоскостью. Отождествим $\mathbb{R}^{2 n}$ с $\mathbb{C}^{n}: p_{k}+i q_{k}=z_{k}$. Путь $\gamma(\varphi)=e^{i \varphi}\{q=0\}, 0 \leqslant \varphi \leqslant \pi / 2$, является искомым. Действительно, построенный путь $\gamma$ положительный, поскольку $\gamma(\varphi)$ - образ под действием фазового потока за время $\varphi$ с гамильтонианом $\sum p_{k}^{2}+q_{k}^{2}$. С другой стороны, путь $\gamma$ - часть положительной петли $l(\varphi)=e^{i \varphi}\{q=0\}, 0 \leqslant \varphi \leqslant \pi$, класс Маслова которой равен $n$ [1].

Следующая теорема - теорема перемежаемости.

Теорема 5.2. Пусть $\Sigma_{1}$ и $\Sigma_{2}$ - обобщенные шлейфы, a $l-n у т ь в$ лагранжевом многообразии Грассмана $\Lambda_{n}$. Тогда

$$
\left|\operatorname{ind}_{\Sigma_{1}}(l)-\operatorname{ind}_{\Sigma_{2}}(l)\right| \leqslant n .
$$

ДокаЗАТЕльСтво. Можно считать, учитывая предложение 4.7, что концы пути $l$ не содержатся в $\Sigma_{1}$ и $\Sigma_{2}$. Поэтому можно допустить, что эти концы трансверсальные лагранжевы плоскости. Замкнем их положительным путем $\gamma$, построенным в лемме 5.1. Согласно предложению 4.5, класс Маслова получившейся петли равен $\operatorname{ind}_{\Sigma_{j}}(l)+\operatorname{ind}_{\Sigma_{j}}(\gamma), j=1,2$. Следовательно, $\operatorname{ind}_{\Sigma_{1}}(l)-$ $\operatorname{ind}_{\Sigma_{2}}(l)=\operatorname{ind}_{\Sigma_{2}}(\gamma)-\operatorname{ind}_{\Sigma_{1}}(\gamma)$. Воспользовавшись оценкой из леммы 5.1 для пути $\gamma$, получаем искомое неравенство.

СлЕДСТВИЕ 5.3. Если лагранжева плоскость эволючионирует под действием Фазового потока с положительно определенньм гамильтонианом, то числа моментов пересечения с любыми двумл обобщенными шлейфами ( с учетом кратностей) различаются на любом отрезке не более чем на число степеней свободы. На отрезке, содержащем $n+1$ моментов пересечения с одним обобщенным шлейбом, найдется момент пересечения с любым другим обобщенным шлейфом.

СлЕДСТВИЕ 5.4. Кратность любой точки обобщенного илейфа $\Sigma \subset \Lambda_{n}$ не превосходит $n$.

Докажем теорему о нулях.

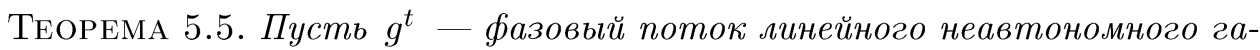
мильтонова векторного поля в $\left(\mathbb{R}^{2 n}, \omega\right), \Sigma$ - обобщенный шлей $\phi, \delta_{i}(i=$ $1,2)$ - лагранжевы плоскости и $g^{t} \delta_{i}=g^{t}\left(\delta_{i}\right), t \in[0,1]$, 一 пути в лагранжевом многообразии Грассмана $\Lambda_{n}$. Тогда

$$
\left|\operatorname{ind}_{\Sigma}\left(g^{t} \delta_{1}\right)-\operatorname{ind}_{\Sigma}\left(g^{t} \delta_{2}\right)\right| \leqslant n .
$$

ДокАЗАТЕльСтво. Можно считать (учитывая предложение 4.7), что лагранжевы плоскости $\delta_{i}, g^{1}\left(\delta_{i}\right)(i=1,2)$ не содержатся в обобщенном шлейфе $\Sigma$ и что плоскости $\delta_{1}$ и $\delta_{2}$ трансверсальны. Соединим плоскости $\delta_{1}$ и $\delta_{2}$ путем $m_{1}$, построенным в доказательстве леммы 5.1. Пути $g^{t} \delta_{1}$ и $m_{1} \cup g^{t} \delta_{2} \cup$ $-g^{1}\left(m_{1}\right)$ гомотопны $\left(-g^{1}\left(m_{1}\right)\right.$ - путь $g^{1}\left(m_{1}\right)$ с противоположной ориентацией). Следовательно, $\operatorname{ind}_{\Sigma}\left(g^{t} \delta_{1}\right)-\operatorname{ind}_{\Sigma}\left(g^{t} \delta_{2}\right)=\operatorname{ind}_{\Sigma}\left(m_{1}\right)-\operatorname{ind}_{\Sigma}\left(g^{1}\left(m_{1}\right)\right)$. 
Имеем $\operatorname{ind}_{\Sigma}\left(g^{1}\left(m_{1}\right)\right)=\operatorname{ind}_{\left(g^{1}\right)^{-1}(\Sigma)}\left(m_{1}\right)$, где $\left(g^{1}\right)^{-1}(\Sigma)$ - обобщенный шлейф. Пользуясь оценкой из леммы 5.1, получаем искомое неравенство.

СЛЕДСТВИЕ 5.6. Если две лагранжевы плоскости эволюиионируют в системе с положительно определенным гамильтонианом, то разность между числами моментов их пересечения ( с учетом кратностей) с обобщенным шлейбом не превосходит $n$.

Обозначим через $g_{H}^{t} \sigma=g_{H}^{t}(\sigma), t \in[0,1]$, путь в лагранжевом многообразии Грассмана $\Lambda_{n}$, образованный эволюцией лагранжевой плоскости $\sigma$ под действием фазового потока гамильтонова векторного поля с гамильтонианом $H\left(g_{H}^{t}\right.$ - отображение фазового потока за время $\left.[0, t]\right)$. Докажем симплектическую версию теоремы сравнения.

ТЕорема 5.7. Если $H_{1} \geqslant H_{0}$, то для обобщенных шлейфов $\Sigma_{1} u \Sigma_{2} u$ лагранжевых плоскостей $\sigma$ и $\lambda$ выполняются неравенства

$$
\begin{aligned}
\operatorname{ind}_{\Sigma_{1}}\left(g_{H_{1}}^{t} \sigma\right) \geqslant & \operatorname{ind}_{\Sigma_{1}}\left(g_{H_{0}}^{t} \sigma\right), \quad \operatorname{ind}_{\Sigma_{1}}\left(g_{H_{1}}^{t} \sigma\right) \geqslant \operatorname{ind}_{\Sigma_{1}}\left(g_{H_{0}}^{t} \lambda\right)-n, \\
& \operatorname{ind}_{\Sigma_{1}}\left(g_{H_{1}}^{t} \sigma\right) \geqslant \operatorname{ind}_{\Sigma_{2}}\left(g_{H_{0}}^{t} \lambda\right)-2 n .
\end{aligned}
$$

ДокАЗАтЕльство. С учетом предложения 4.7 можно считать, что плоскости $\sigma, g_{H_{1}}^{1}(\sigma), g_{H_{0}}^{1}(\sigma), \lambda, g_{H_{1}}^{1}(\lambda), g_{H_{0}}^{1}(\lambda)$ не содержатся в обобщенных шлейфах $\Sigma_{1}$ и $\Sigma_{2}$. Таким образом, теорему достаточно доказать для случая $H_{1}>H_{0}$. Рассмотрим гомотопию $H_{m}=(1-m) H_{0}+m H_{1}$ гамильтонианов.

ЛЕмма 5.8. Путь $g_{H_{m}}^{1} \sigma=g_{H_{m}}^{1}(\sigma), m \in[0,1]$, положительньии.

Продолжим доказательство теоремы. Пути $g_{H_{0}}^{t} \sigma \cup g_{H_{m}}^{1} \sigma$ и $g_{H_{1}}^{t} \sigma$ гомотопны; значит (согласно следствию 1.2), $\operatorname{ind}_{\Sigma_{1}}\left(g_{H_{1}}^{t} \sigma\right) \geqslant \operatorname{ind}_{\Sigma_{1}}\left(g_{H_{0}}^{t} \sigma\right)$. Первое неравенство теоремы доказано. Имеем $\operatorname{ind}_{\Sigma_{1}}\left(g_{H_{1}}^{t} \sigma\right) \geqslant \operatorname{ind}_{\Sigma_{1}}\left(g_{H_{1}}^{t} \lambda\right)-n$ согласно теореме 5.5. Это вместе с первым неравенством доказывает второе неравенство. Пользуясь теоремой 5.2 , получаем, что $\operatorname{ind}_{\Sigma_{1}}\left(g_{H_{1}}^{t} \sigma\right) \geqslant \operatorname{ind}_{\Sigma_{2}}\left(g_{H_{1}}^{t} \sigma\right)-n$, а это вместе со вторым неравенством доказывает третье неравенство.

ДокАЗАТЕЛЬСтво ЛЕммы 5.8. Лемма 5.8 вытекает из следующего утверждения:

Пусть неавтономный квадратичный гамильтониан $H$ зависит от параметра $s$, так что $\widetilde{H}=d H / d s>0$. Тогда семейство симплектоморфизмов $\psi_{s}=g_{H(s)}^{1}\left(g_{H(0)}^{1}\right)^{-1}$ задается положительно определенным гамильтонианом.

Производная решения $(p(t, s), q(t, s))$ уравнений Гамильтона $\dot{p}=-H_{q}$, $\dot{q}=H_{p}$ по параметру $s$ (обозначим ее через $\left(h_{1}(t, s), h_{2}(t, s)\right)$ ) удовлетворяет следующей системе уравнений в вариациях:

$$
\dot{h}_{1}=-\frac{\partial^{2} H}{\partial q \partial p} h_{1}-\frac{\partial^{2} H}{\partial q \partial q} h_{2}-\frac{\partial \widetilde{H}}{\partial q}, \quad \dot{h}_{2}=\frac{\partial^{2} H}{\partial p \partial p} h_{1}+\frac{\partial^{2} H}{\partial p \partial q} h_{2}+\frac{\partial \widetilde{H}}{\partial p}
$$

с начальными условиями $h_{1}(0, s)=0, h_{2}(0, s)=0$.

По теореме Эйлера $\omega\left(x, v_{K}(x)\right)=2 K(x)$ для гамильтонова векторного поля $v_{K}$ с гамильтонианом $K$. Таким образом, мы должны показать, что 
$\omega\left((p(1, s), q(1, s)),\left(h_{1}(1, s), h_{2}(1, s)\right)\right)>0$. Имеем

$$
\begin{aligned}
\frac{\partial}{\partial t} \omega( & \left.(p(t), q(t)),\left(h_{1}(t), h_{2}(t)\right)\right)=\dot{p} h_{2}-\dot{q} h_{1}+p \dot{h}_{2}-q \dot{h}_{1} \\
= & -\frac{\partial H}{\partial q} h_{2}-\frac{\partial H}{\partial p} h_{1}+p\left(\frac{\partial^{2} H}{\partial p \partial p} h_{1}+\frac{\partial^{2} H}{\partial p \partial q} h_{2}+\frac{\partial \widetilde{H}}{\partial p}\right) \\
& +q\left(\frac{\partial^{2} H}{\partial q \partial p} h_{1}+\frac{\partial^{2} H}{\partial q \partial q} h_{2}+\frac{\partial \widetilde{H}}{\partial q}\right)
\end{aligned}
$$

(мы опускаем параметр $s$, точка сверху означает производную по $t$ ). Далее,

$$
\frac{\partial H}{\partial p}=p \frac{\partial^{2} H}{\partial p \partial p}+q \frac{\partial^{2} H}{\partial q \partial p}, \quad \frac{\partial H}{\partial q}=p \frac{\partial^{2} H}{\partial p \partial q}+q \frac{\partial^{2} H}{\partial q \partial q},
$$

так как $\partial H / \partial p$ и $\partial H / \partial q$ однородны степени 1. Следовательно, используя теорему Эйлера, получаем

$$
\frac{\partial}{\partial t} \omega\left((p(t), q(t)),\left(h_{1}(t), h_{2}(t)\right)\right)=p \frac{\partial \widetilde{H}}{\partial p}+q \frac{\partial \widetilde{H}}{\partial q}=2 \widetilde{H}>0 .
$$

Интегрированием, с учетом начальных условий, устанавливаем нужное неравенство. Лемма доказана.

Пусть функции Гамильтона положительно определены.

СлЕдСТвиЕ 5.9. На отрезке, содержащем $n+1$ моментов пересечения лагранжевой плоскости, эволюиионирующей в одной гамильтоновой системе, с обобщенным шлейфом, найдется хотя бы один момент пересечения лагранжевой плоскости, эволючионирующей в системе с большей (нестрого) функиией Гамильтона, с тем же илейфом. Более того, разность между числами моментов пересечения ( с учетом кратностей) для систем с меньшей и большей функциями Гамильтона не превосходит $n$.

ЗАмечАниЕ. Все оценки теорем этого параграфа точны. Для любого неравенства любой теоремы можно подобрать обобщенные шлейфы (даже с заранее заданным числом матриц, по которым строится обобщенный шлейф), пути в лагранжевом грассманиане, лагранжевы плоскости и гамильтонианы так, что неравенства обратятся в равенства. Интересно, что ни в одном известном автору доказательстве теорем Штурма не используется геометрия расположения двух (обобщенных) шлейфов, - например, на сколько частей они делят лагранжев грассманиан (шлейфы двух трансверсальных лагранжевых плоскостей делят лагранжев грассманиан на $n+1$ частей), что для обобщенных шлейфов и неизвестно.

\section{ЛитературА}

1. Арнольд В. И. О характеристическом классе, входящем в условие квантования. Функц. анализ и его прил., 1, вып. 1, 1-14 (1967).

2. Арнольд В. И. Теоремы Штурма и симплектическая геометрия. Функц. анализ и его прил., 19, вып. 4, 1-10 (1985).

3. Арнольд В. И. Обыкновенные дифференциальные уравнения. Наука, М., 1984. 
4. Bott $R$. On the iterations of closed geodesics and the Sturm intersection theory. Commun. Pure Appl. Math., 9, No. 2, 171-206 (1956).

5. Edwards H. M. A generalised Sturm theorem. Ann. Math., 80, No. 1, 22-57 (1964).

6. Gärding L. An inequality for hyperbolic polynomials. J. Math. Mech., 8, No. 6, 957-965 (1959).

7. Гивенталь А. Б. Теорема Штурма для гиперэллиптических интегралов. Алгебра и анализ, 1, вып. 5, 95-102 (1989).

8. Givental A. B. Nonlinear generalization of the Maslov index. Adv. Sov. Math., Vol. 1, 1990, pp. 71-103.

9. Лидский B. Б. Осцилляционные теоремы для канонической системы дифференциальных уравнений. ДАН СCCP, 102, No. 5, 877-880 (1955).

10. Morse $M$. A generalization of the Sturm theorems in $n$-space. Math. Ann., 103, 52-69 (1930).

11. Morse $M$. The calculus of variation in the large. In: AMS Coll. Publ., Vol. 18, New York, 1934, pp. 80-106.

12. Robbin J., Salamon D. The Maslov index for paths. Topology, 32, No. 4, 827-844 (1993).

13. Sturm C. Memoire sur les equaitions differentielles du second ordre. J. Math. Pures Appl., 1, 106-186 (1836).

14. Хованский A. Г. Аналоги неравенства Александрова-Фенхеля для гиперболических форм. ДАН СССР, 274, № 4, 1332-1334.

15. Якубович B. A. Аргументы на группе симплектических матриц. Матем. сб., 55, вып. 3, 255-280 (1961).

16. Якубович $B$. A. Осцилляторные свойства решений канонических уравнений. Матем. сб., 56, вып. 1, 3-42 (1962).

17. Якубович $B$. A. Неосцилляторность нелинейных периодических уравнений и смежные вопросы. Алгебра и анализ, 3, вып. 5, 229-253 (1991).

Московский государственный университет им. М. В. Ломоносова, механико-математический факультет
Поступило в редакцию 23 декабря 1997 г. 